\title{
LOS PLECÓPTEROS DE GALICIA (ESPAÑA): DISTRIBUCIÓN ALTITUDINAL Y PERIODOS DE VUELO
}

\author{
P. Membiela \\ Instituto B. «Otero Pedrayo». P. Feijoo, 14. 32005 Orense. Spain. \\ Palabras clave: altitudinal distribution, flight penods, Plecoptera, Galicia, Spain.
}

\begin{abstract}
THE STONEFLIES OF GALICIA: ALTITUDINAL DISTRIBUTION AND FLIGHT PERIODS

This work is an analysis of the flight periods and the altitudinal distnbution of stoneflies in Galicia (NW Iberian Peninsula) based on the capture of imagos, carried out between 1979 and 1985 in 60 localities throughout Galicia. The main conclusions in relation with the altitudinal distribution are: firstly, the absence of taxa that only appear in middle altitudes (500-1000 m), secondly, the biggest group is the one with large altitudinal distnbution and thirdly, there are some taxa which are found only in high or low altitudes. We can also observe how the majonty of species show a spring-summer or autumnal flight penod, while a few taxa have a winter flight period.
\end{abstract}

\section{INTRODUCCIÓN}

El estudio de los períodos de vuelo y la distribución altitudinal representan dos aproximaciones clásicas al conocimiento del comportamiento biológico y ecológico de los plecópteros. Este tipo de trabajos ha constituido durante mucho tiempo la aportación fundamental al estudio de su ciclo vital y de los factores de distribución. La complejidad del ciclo vital $\left(\mathbf{n}^{\circ}\right.$ puestas, $\mathbf{n}^{\circ}$ huevos/puesta, periodo de eclosión, crecimiento larval, periodo de emergencia, duración de la vida imaginal, posibles diapausas) hace muy difícil y laborioso su conocimiento en profundidad. Por tanto, continuan teniendo gran interés los datos sobre los periodos de vuelo y distribución altitudinal, pues aún representan en muchos casos la única información existente o la más fiable sobre el ciclo vital.

En la Península Ibérica (Pirineos incluidos) los datos que poseemos sobre los períodos de vuelo y la distribución altitudinal son limitados; los tra-

Limnetica, 6: 131-136 (1990)

(C) Asociación Española de Limnología, Madrid, Spain bajos de Aubert (1963) sobre el conjunto de la Península y Berthelemy (1966) sobre los Pirineos constituyen las aportaciones fundamentales sobre ambos temas. Otros trabajos importantes son los de Puig (1984) sobre la distribución altitudinal de los plecópteros en Cataluña y el de Sánchez ORTEGA (1986) sobre los períodos de emergencia y distribución altitudinal de los plecópteros de Sierra Nevada.

En Galicia, hasta el momento sólo ha sido publicado un trabajo que recoge distribución altitudinal y los períodos de vuelo de los plecópteros de la cuenca del Tambre (Membiela, 1987). En el presente estudio se recogen y analizan datos sobre los períodos de vuelo e influencia de la altitud en los plecópteros del país gallego.

\section{MATERIAL Y MÉTODOS}

Entre 1979 y 1985 se ha llevado a cabo un estudio sobre los plecópteros de Galicia, basado 
fundamentalmente en las capturas de adultos realizadas en 60 estaciones. De ellas, 40 han sido muestreadas periódicamente al menos de forma trimestral durante un ano, mientras en las restantes 20 estaciones sólo se han realizado capturas esporádicas de adultos. En Membiela (1988, en prensa) puede encontrarse una descripción de las estaciones, períodos y métodos de muestreo.

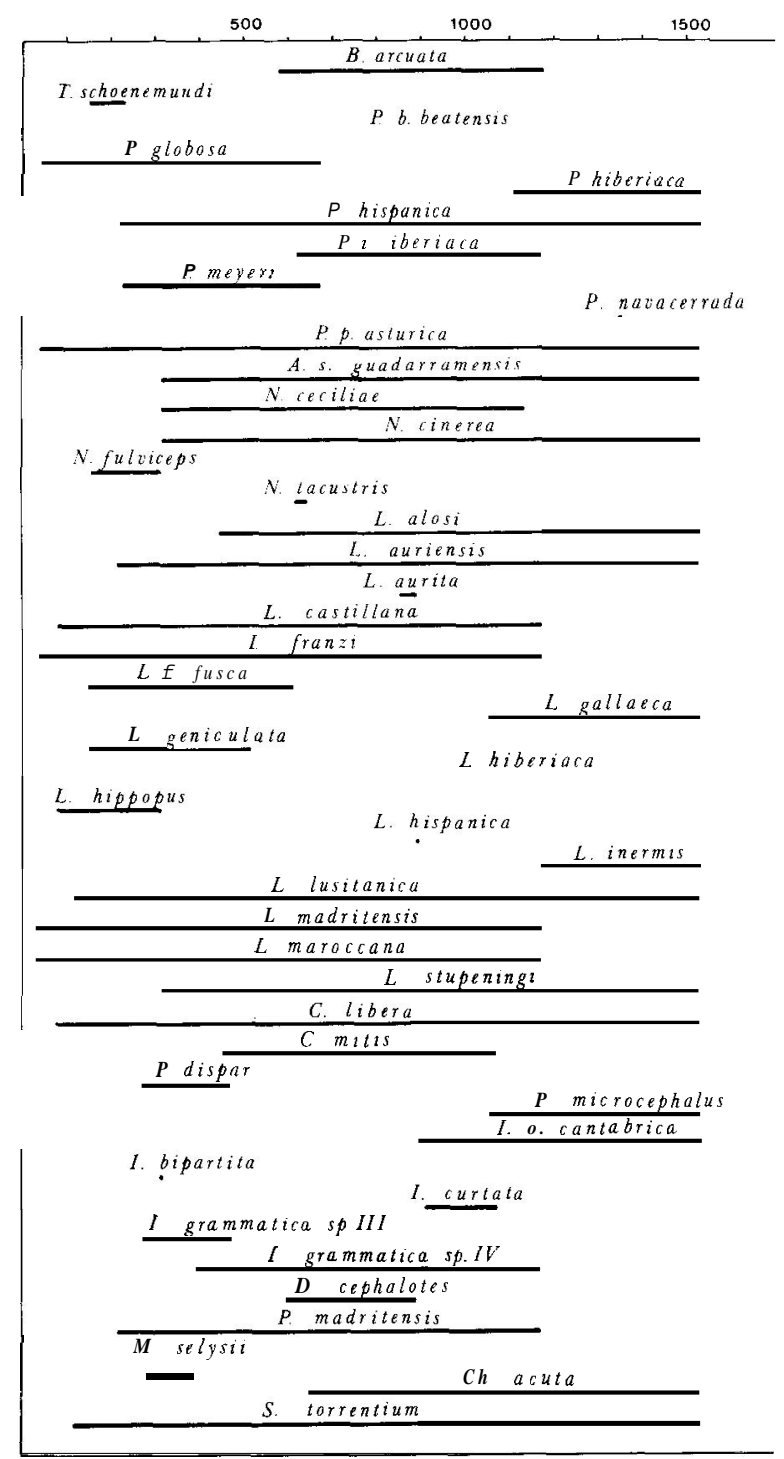

Figura 1.- Distribución altitudinal de los plecópteros en Galicia.

Altitudinal distnbution of stoneflies in Galicia.

\section{RESULTADOS}

En las figs. 1 y 3 se resumen gráficamente la distribución altitudinal y los períodos de vuelo de los diferentes taxones encontrados en Galicia, deducidos fundamentalmente de nuestras capturas (ver Membiela, en prensa) y dónde se incluyen los datos previamente publicados sobre la cuenca del Tambre (Membiela, 1987). Asimismo, hemos recogido las escasas citas fiables suministradas por otros autores (AUBERT, 1961, 1962, 1963; Zwick, 1972, 1982; BeRTHELEMY \& TERRA, 1980).

\section{DISCUSIÓN}

\section{Distribución altitudinal}

A pesar de las imprecisiones que presenta la distribución altitudinal observada para los diferentes taxones, fundamentalmente en razón de su frecuencia y/o abundancia, el presente estudio permite conocer con relativa profundidad el comportamiento de los plecópteros en Galicia en relación con la altitud.

A partir de los datos que se resumen gráficamente en la fig. 1, podemos señalar la existencia de 5 tipos de distribución altitudinal y atribuirles las especies registradas (Leuctra besucheti Aubert 1962 no se ha calificado por ser cita dudosa):

1. Taxones propios de zonas bajas (7): Taeniopteryx schoenemundi (Mertens, 1923), Leuctra geniculata Stephens, 1835, L. hippopus Kempny, 1899, Perlodes dispar Rambur, 1842, Isoperla bipartita Aubert, 1962, I. grammatica sp. III sensu Berthélemy, 1979 y Marthamea selysii (Pictet, 1841).

2. Taxones de zonas bajas-medias (3): Protonemura globosa Berthélemy $\boldsymbol{y}$ Terra, 1980, P. meyeri (Pictet, 1841) y Leuctra fusca fusca (Linné, 1758).

\footnotetext{
* Taxones clasificados de forma provisional en una categoría determinada, debido a nuestros escasos datos y/o los existentes de otros autores sobre la Península Ibénca.

** Taxones clasificados de forma provisional en una categoría determinada, debido a nuestros escasos datos y/o los existentes de otros autores sobre la Península Ibénca.
} 
3. Taxones de zonas medias-altas (7): Brachyptera arcuata (Klapálek, 1902), Protonemura intricata iberiaca Zwick, 1972, Leuctra alosi Navás, 1919, Zsoperla grammatica sp. N sensu Berthélemy, 1979, Chloroperla acuta Berthélemy y Terra, 1980, Protonemura beatensis beatensis (Despax, 1929)* e Zsoperla curtata Navás, 1924*.

4. Taxones de zonas altas (7): Protonemura hiberiaca Aubert, 1963, P. navacerrada Aubert, 1954, Leuctra gallaeca Membiela, 1989, L. hiberiaca Aubert, 1956, L. inermis Kempny, 1899, Perlodes microcephalus (Pictet, 1841) e Zsoperla acicularis cantabrica Aubert, 1956.

5. Taxones de amplia distribución altitudinal (21): Protonemura hispanica Aubert, 1956, P . pyrenaica asturica Aubert, 1954, Amphinemura sulcicollis guadarramensis Aubert, 1952, Nemoura cinerea (Retzius, 1783), Leuctra auriensis Membiela, 1989, L. castillana Aubert, 1956, L. franzi Aubert, 1956, L. lusitanica Aubert, 1962, L. madritensis Aubert, 1952, L. maroccana Aubert, 1956, L. stupeningi Illies, 1954, Capnioneura libera (Navás, 1909), Perla madritensis Rambur, 1842, Siphonoperla torrentium Pictet, 1841, Nemoura ceciliae Aubert, 1956*, N. fulviceps Klapálek, $1902^{*}, N$. lacustris E. Pictet, $1865^{*}$, Leuctra aurita Navás, 1919*, L. hispanica Aubert, 1952*, Capnioneura mitis Despax, 1932* y Dinocras cephalotes (Curtis, 1827)*.

El criterio seguido ha sido considerar zonas bajas entre 0-500 m, zonas medias entre 500-1000 m y altas más de $1000 \mathrm{~m}$. No obstante, esta clasificación trata de definir diferentes tipos de distribución y, como es obvio, en algunos casos resulta problemática la asignación de un taxón determinado a uno u otro grupo.

Para el caso concreto de este estudio, después del análisis de la distribución altitudinal se pueden deducir algunas conclusiones. En primer lugar, la ausencia de taxones exclusivos de zonas medias $(500-1000 \mathrm{~m})$. En segundo lugar, que el grupo más numeroso, con diferencia, está representado por las especies que muestran una amplia distribución altitudinal. Por último, la existencia de una serie de taxones confinados a un estrecho margen de altitudes, bien en zonas altas o en zonas bajas.

Otros autores (AUBERT, 1946; KAMLER, 1967) han señalado que aparecen unos taxones con am-

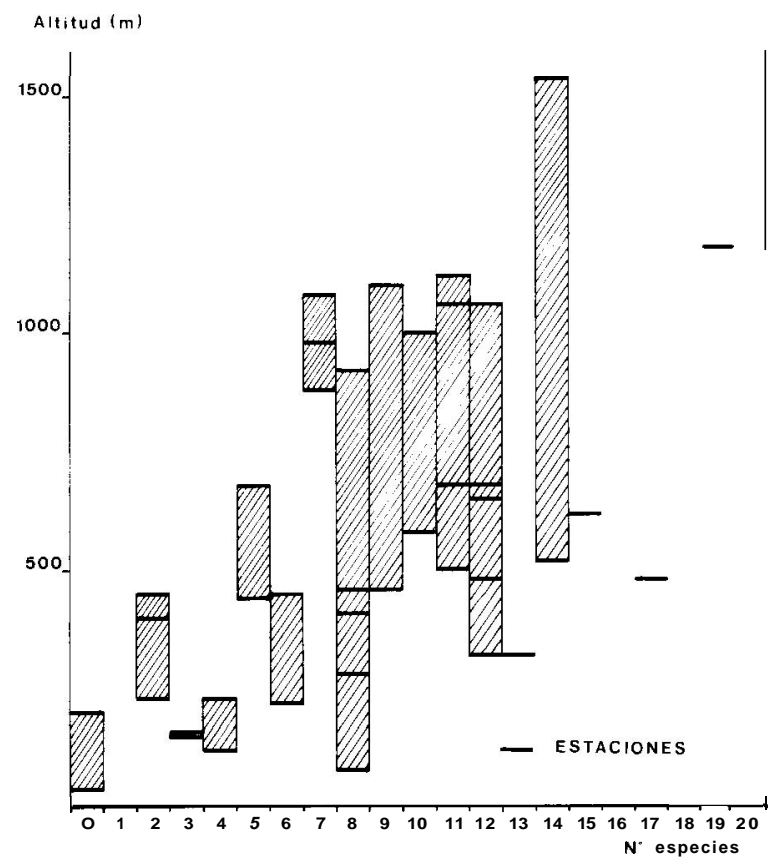

Figura 2.- Relación entre altitud y riqueza específica para las 40 estaciones (est. 1 a 40) muestreadas periódicamente.

Relation between altitude and number of species for the 40 localities periodically sampled.

plia distribución altitudinal y otros limitados a una gama determinada. En tal sentido, la altitud ha sido reconocida como uno de los factores que pueden explicar la distribución de los plecópteros (Hynes, 1941; Aubert, 1946; Knight \& Gaufin, 1966; W ARD, 1982).

También se ha estudiado la posible relación existente entre altitud y riqueza específica (fig. 2). En nuestro caso, puede achacarse a los datos presentados una cierta falta de homogeneidad, aunque ello no es suficiente para desvirtuar la clara relación observada entre la altitud de una estación y el número de especies presentes en la misma. En líneas generales existe una tendencia al incremento de este parámetro desde las zonas más bajas hasta las localidades próximas a los $500 \mathrm{~m}$. La mayor riqueza específica suele darse entre 500-1200 m de altitud. Estas conclusiones están de acuerdo con la afirmación de que la riqueza específica y la diversidad son menores en las zonas bajas o muy altas. Así, Aubert (1946) indica que en Suiza el mayor número de especies aparece sobre 
$1000 \mathrm{~m}$ de altitud, mientras que KaMLer (1967), en su estudio de los plecópteros de algunos macizos montañosos de Polonia, señala que la mayoría de las especies han sido encontradas entre 800 y $1700 \mathrm{~m}$ y que es en esta zona donde también se presentan las mayores densidades. En Galicia no se observa un descenso claro del número de especies en las zonas más elevadas tal como ocurre en otras áreas (ver AUBert, 1946; BERTHELEMY, 1966), puesto que no se alcanzan las altitudes necesarias (al menos $2000 \mathrm{~m}$ ) para que este fenómeno se manifieste claramente.

\section{Períodos de vuelo}

A pesar del grado de imprecisión inherente a este tipo de estudios, se puede afirmar que el presente análisis da una imagen bastante aproximada de la distribución temporal de los plecópteros adultos en Galicia.

Nuestra intención desde un principio ha sido incluir los diferentes períodos de vuelo en una serie de grupos o categorías, tal como han realizado otros autores. No obstante, resulta difícil clasificar una realidad relativamente compleja y en algunos casos la inclusión de un taxón en uno u otro grupo es problemática.

A partir de los datos que se resumen gráficamente en la fig. 3 , hemos establecido para el conjunto de Galicia las siguientes categorías (se considera como primavera los meses III-IV-V, verano los meses VI-VII-VIII, otoño los meses IX-XXI e invierno los meses XII-I-II):

1. Taxones con períodos de vuelo de larga duración (3): P. globosa, que se extiende por todas las estaciones del año. N. ceciliae ${ }^{* *}$ y $N$. fulviceps $^{* *}$, presentan un periodo largo pero no aparecen en el invierno como adultos.

2. Taxones primaverales (3): B. arcuata, L. hippopus y $P$. dispar**.

3. Taxones primaverales-estivales (12): P. p. asturica, A. s. guadarramensis, $N$. cinerea, L. inermis, P. microcephalus, I. grammatica sp. III, $P$. madritensis, S. torrentium, P. navacerrada**, N. lacustris ${ }^{* *}$, I. bipartita**, D. cephalotes ${ }^{* *}$.

4. Taxones estivales (6): P. i. iberiaca, I. a. cantabrica, I. curtata, I. grammatica sp. $N, M$. selysii y $C$. acuta.

5. Taxones estivales-otoñales (3): L. alosi, L. franzi y L. aurita.
6. Taxones otoñales (13): P. b. beatensis, $\boldsymbol{P}$. hiberiaca, $P$. hispanica, L. auriensis, $L$. besucheti, L. castillana, L. f. fusca, L. gallaeca, L. geniculata, L. hiberiaca, L. hispanica, L. lusitanica y L. madritensis.

7. Taxones invernales (3): L. maroccana, L. stupeningi y $C$. libera.

8. Taxones invernales-primaverales (2): P. meyeri y $C$. mitis.

Del análisis del número de taxones que pertenecen a cada grupo se deduce que la mayoría de las especies presentan un periodo de vuelo primaveral-estival-u otoñal, mientras que son muy pocas las que emergen durante el-invierno. Nuestras conclusiones concuerdan con HyNes (1976),

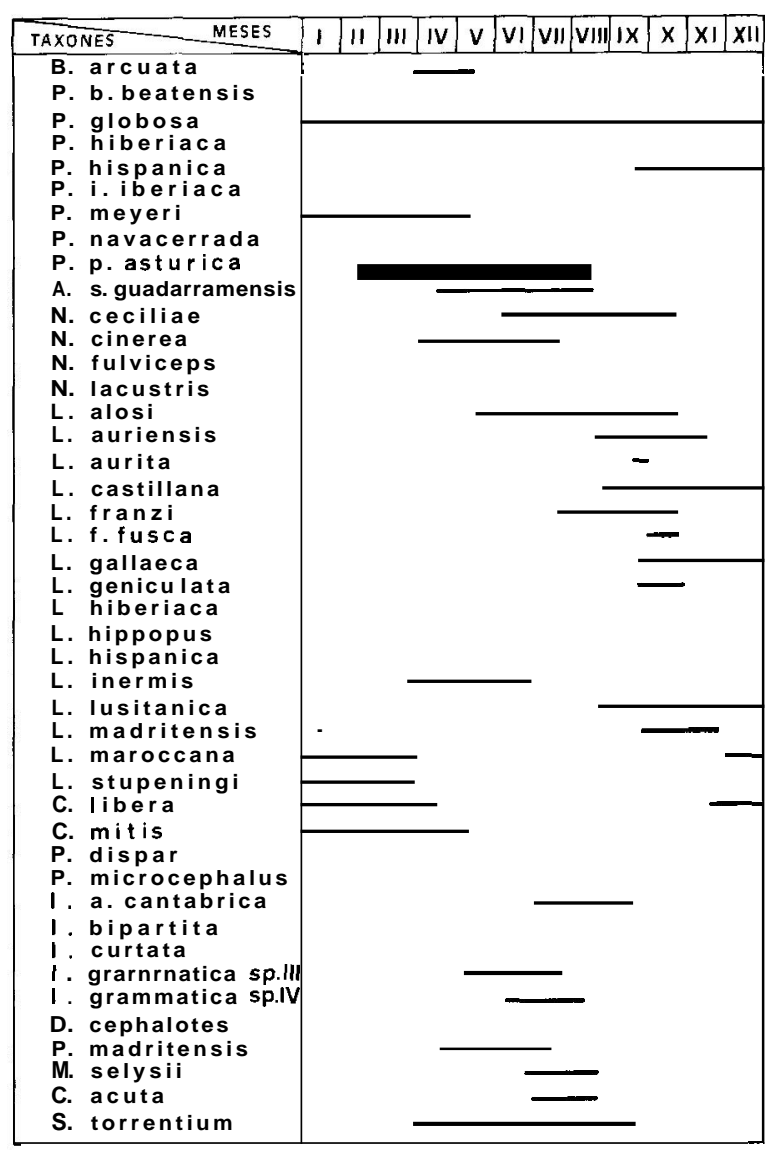

Figura 3.- Periodos de vuelo de los plecópteros en Galicia. Flight periods of stoneflies in Galicia. 
quien en su revisión de la biología del grupo señala como en las latitudes medias del hemisferio norte la mayoría de las especies emergen en una época generalmente corta del año, mientras unas pocas especies vuelan durante varios meses; en el mismo sentido BERTHELEMY (1966) indica la existencia en altitudes bajas de dos grandes periodos de captura de adultos en primavera y otoño separados por un mínimo en verano. Por el contrario, sólo en un caso (P. globosa) hemos observado la posible relación existente entre la amplitud del periodo de vuelo y la distribución ecológica (ver BERTHELEMY, 1966), explicada como una tolerancia amplia única a los factores del medio.

\section{AGRADECIMIENTOS}

Este trabajo ha sido realizado gracias a la subvención concedida por la CAZCYT, proyecto de investigación PB86-0406.

\section{Bibliography}

Aubert, J., 1946. Les Plécopteres de la Suisse Romande. Mitt. schweiz. ent. Ges. 20: 8-128.

Aubert, J., 1961. Los Plecópteros del Instituto Español de Entomología. Graellsia 19: 91-94.

AUBERT, J., 1962. Quelques Leuctra nouvelles pour l'Europe. Mitt. schweiz. ent. Ges. 35: 155-169.

Aubert, J., 1963. Les Plécopteres de la Péninsule Ibérique. Eos 39: 23-107.
Berthelemy, C., 1966. Recherches écologiques et biogéographiques sur les Plécopteres et Coléoptères d'eau courante (Hydraena et Elminthidae) des Pyrenées. Annls. Limnol. 2 (2): $227-458$.

Berthelemy, C. \& L.S.W. Terra, 1980. Plécopteres du Portugal. Annls. Limnol. 16 (2): 159-182.

HyNES, H.B.N., 1941. The taxonomy and ecology of the nymphs of the British Plecoptera with notes on the adults and eggs. Trans. Roy. Ent. Soc., Londres, 91: 459-557.

Hynes, H.B.N., 1976. Biology of Plecoptera. Ann. Rev. Entom., Palo Alto, 21: 135-153.

KAMLER, E., 1967. Distribution of Plecoptera and Ephemeroptera in relation to the altitude above mean sea level and current speed in mountain waters. Pol. Arch. Hydrobiol. 14 (27): 29-42.

KNIGHT, A.W. \& A.R. Gaufin, 1966. Altitudinal distribution of stoneflies (Plecoptera) in a Rocky Mountain drainage system. J. Kans. Entomol. Soc. 39: 668-675.

Membiela, P., 1987. Primera contribución al conocimiento de los Plecópteros de Galicia: La cuenca del Tambre. Limnetica 1: 197-202.

Membiela, P., 1988. Los Plecópteros de Galicia: estudio taxonómico y ecológico. Tesis doctoral. Universidad de Santiago. Santiago de Compostela. 399 p.

MEMBiela, P. (en prensa). Contribución al conocimiento faunístico de los Plecópteros de Galicia (Plecoptera). Bol. Asoc. esp. Entomol.

Puig, M.A., 1984. Distribution and ecology of the stoneflies (Plecoptera) in Catalonian rivers (N.E. Spain). Annls. Limnol. 20 (1-2): 75-79.

SÁnchez ORTEgA, A., 1986. Taxonomia, ecología y ciclos de vida de los Plecópteros de Sierra Nevada. Tesis Doctoral. Universidad de Granada. 362 p.

WARD, J.V., 1982. Altitudinal zonation of Plecoptera in a Rocky Mountain stream. Aquatic Insects 4 (2): 105-110.

ZwICK, P., 1972. Plecoptera (Ins.) aus dem Mittelmeergebiet, vor allem aus Portugal und Spanien. Ciênc. biol. 1: 7-17.

ZwICK, P., 1982. The stonefly collection of F. Klapálek in Prague, with notes on the Nemouridae (Plecoptera). Aquatic Znsects 4: 39-48. 